\title{
Bisphosphonates for osteoarthritis prevention: “Holy Grail" or not?
}

\section{Kenneth G Saag}

The search for effective disease modifying osteoarthritis (OA) agents has been a long and winding road with much unrealised promise. While a debate persists about whether OA is predominately a disease of bone, cartilage, or both, there has been interest in the possible OA disease modifying effects of bone antiresorptive agents including oestrogens, selective oestrogen receptor modulators, calcitonin and bisphosphonates. ${ }^{1}$ Bisphosphonates, the most commonly used therapeutic agents for osteoporosis prevention and treatment, hold OA treatment appeal based on their known pharmacology of altering bone remodelling through a direct inhibitory effect on the osteoclast. Such effects could retard subchondral bone remodelling, believed important by some in osteophyte formation and subchondral sclerosis. Also of potential benefit in OA, bisphosphonates have effects beyond the osteoclast and may be slightly immunomodulating via inhibition of pro-inflammatory cytokines. ${ }^{2}$ In vitro, etidronate binds to human cartilage ${ }^{3}$ and can modestly inhibit matrix metalloproteinases. ${ }^{4}$ Neridronate stimulated osteoblasts to produce osteocalcin in an OA model, providing yet another potential pathway for bisphosphonate activity in OA. ${ }^{5}$ Animal data supports a possible reduction in osteophyte progression and a suppression in subchondral bone resorption with bisphosphonates. Rabbits treated with zoledronic acid $^{6}$ and rats receiving alendronate $^{7}$ experienced chondroprotection. Bisphosphonates may also stimulate collagen and proteoglycan synthesis. ${ }^{89}$ Carbone and colleagues examined women in the Health, Aging, and Body Composition Study, observing a reduction in magnetic resonance imagery (MRI)associated marrow oedema in subchondral bone but no reduction in knee OA

\footnotetext{
Correspondence to: Kenneth G Saag, Center for Education and Research on Therapeutics (CERTs) of Musculoskeletal Disorders, Division of Clinical Immunology and Rheumatology, University of Alabama at Birmingham, 820 Faculty Office Tower, 510 20th Street South, Birmingham, Alabama 35294-3708, USA;

Kenneth.Saag@ccc.uab.edu
}

radiographic progression among those receiving alendronate. ${ }^{10}$ Cohort studies of this type are limited potentially by systematic differences in OA predilection among those who do and do not receive bisphosphonates. Randomised controlled trials of risedronate initially suggested a bisphosphonate OA benefit in terms of pain, function, radiographic joint space changes and retained vertical trabecular structure. Spector and colleagues found a reduction in the Western Ontario and MacMaster Universities (WOMAC) OA index, along with a non-significant trend towards reduced joint space narrowing in patients receiving three times the usual osteoporosis dose of risedronate $(n=284$ patients). ${ }^{11}$ However, a subsequent much larger randomised controlled trial ( $n=2483$ patients) did not demonstrate a clear OA advantage with respect to radiographic joint space narrowing or WOMAC subscales; however, it was observed that the biochemical measure of cross-linking telopeptide of type II collagen was reduced compared to placebo. ${ }^{12}$ A subset analysis of the study population who were "rapid joint space losers" and who were randomised to risedronate experienced more retained vertebral trabecular structure (particularly at a $50 \mathrm{mg} /$ week risedronate dose) than those receiving placebo. ${ }^{13}$ Some have speculated that this larger clinical trial was underpowered based on a low rate of OA progression in the placebo group. Further, the timing of potential benefit of antiresorptives in OA may be critical with the greatest benefit seen very early in the disease course, although longerterm follow-up for late effects is also missing in many clinical trials. Lastly, it should be noted that independent of their effects on spinal fractures, several bisphosphonates have reduced low back pain. This finding raises questions about whether this effect alternatively could be mediated by a reduction in spinal OA progression.

In this issue of Annals of the Rheumatic Diseases, Neogi and colleagues (see page 1427) report on a cleverly conducted secondary analysis of 200 patients randomly selected from the large and well known Fracture Intervention Trial (FIT). ${ }^{14}$ When radiographs were carefully read against a standard atlas they found a small reduction in spinal osteophyte progression in those patients randomised to receive alendronate compared to placebo. While the spinal radiographic changes were significant, they were subtle and the authors acknowledged that this level of radiographic difference may be of minimal clinical relevance. Similar benefits were seen in terms of disc space narrowing in the lumbar spine, although the significance of this finding did not persist when the thoracic spine was included in the analysis. Although this secondary analysis of FIT was very carefully conducted, there was only modest correlation between the radiograph readers, further questioning the clinical relevance of the findings. Overall, the radiographic reading methods used should have resulted in a non-differential outcome misclassification between those receiving and not receiving alendronate. This study was limited by the fact that the results may not generalise to those without osteoporosis. Since OA occurs preferentially among those without osteoporosis, most studies of OA have been conducted in these patients.

Does this paper support a stronger role for bone in the pathogenesis of osteoarthritis or for bisphosphonates as agents that may retard OA progression? Are the effects of bisphosphonates differential and, if so, could the alendronate used in this study be more efficacious than the very equivocal (likely negative) evidence supporting risedronate as an OA therapy? These and other important clinical questions cannot be fully answered by this paper or by the existing literature, but the results from this important study by Neogi and colleagues provide clear further rationale for additional large-scale comparative clinical trials of bisphosphonates and other bone antiresorptive agents in OA. Since many osteoporosis studies obtain serial spinal radiographs, it would be prudent that this information be analysed as a possible osteoarthritis endpoint. Even if it can be much more consistently demonstrated that all or certain bisphosphonates reduce OA progression in the spine, it is not at all clear that this will correlate with benefits at the knee or hip; the key anatomic areas that lead to the most OA morbidity. In addition to the need for these further studies evaluating bisphosphonate efficacy OA, a recent spate of mostly uncontrolled observations suggest possible new bisphosphonate safety issues. 
One such potential safety signal of exposure to bisphosphonates with long skeletal retention is oversuppression of bone turnover leading to a proposed increased rate of atypical insufficiency fractures. ${ }^{15}$ So far, the evidence justifying this and other newer safety signals such as atrial fibrillation is rather limited, but additional pharmacoepidemiological studies on these topics will allow for eventual better determination of a bisphosphonate benefit-risk ratio. Pending these now more urgently needed further studies on OA efficacy and longer-term safety with bisphosphonate, when an osteoporosis therapy is otherwise needed, a potential beneficial effect on OA might provide slight added value in the decision to prescribe a bisphosphonate.

Competing interests: KGS has been a consultant or speaker for Novartis, Merck, Lilly, Angen, Proctor \& Gamble and Roche.

Accepted 13 July 2008

Ann Rheum Dis 2008:67:1358-1359.

doi:10.1136/ard.2008.089912

\section{REFERENCES}

1. Abramson SB, Honig S. Antiresorptive agents and osteoarthritis: more than a bone to pick? Arthritis Rheum 2007;56:2469-73.
2. Frith JC, Monkkonen J, Auriola S, Monkkonen H, Rogers MJ. The molecular mechanism of action of the antiresorptive and antiinflammatory drug clodronate: evidence for the formation in vivo of a metabolite that inhibits bone resorption and causes osteoclast and macrophage apoptosis. Arthritis Rheum

2001;44:2201-10.

3. Evans CH, Mears DC. Binding of the bone-seeking agent 99mTc-1-hydroxyethylidene-1,1-diphosphonic acid to cartilage and collagen in vitro and its stimulation by Er3+ and low pH. Calcif Tissue Int 1980;32:91-4

4. Edmonds-Alt X, Breliere JC, Roncucci R. Effects of 1-hydroxyethilene-1,1-diphosphonate and (chloro-4phenyl)-thiomethylene bisphosphonic acid (SR 41319) on the mononuclear cell factor-mediated release of neutral proteinases by articular chondrocytes and synovial cells. Biochem Pharmacol 1985;34:4043-9.

5. Corrado A, Cantatore FP, Grano M, Colucci S. Neridronate and human osteoblasts in normal osteoporotic and osteoarthritic subjects. Clin Rheumatol 2005;24:527-34.

6. Podworny NV, Kandel RA, Renlund RC, Grynpas MD Partial chondroprotective effect of zoledronate in a rabbit model of inflammatory arthritis. J Rheumatol 1999;26:1972-82.

7. Hayami T, Pickarski M, Wesolowski GA, McLane J, Bone A, Destefano J, et al. The role of subchondral bone remodeling in osteoarthritis: reduction of cartilage degeneration and prevention of osteophyte formation by alendronate in the rat anterior cruciate ligament transection model. Arthritis Rheum 2004;50:1193-206

8. Guenther HL, Guenther HE, Fleisch $H$. The effects of 1-hydroxyethane-1,1-diphosphonate and dichloromethanediphosphonate on collagen synthesis by rabbit articular chondrocytes and rat bone cells. Biochem J 1981;196:293-301.
9. Muehleman C, Green J, Williams JM, Kuettner KE, Thonar EJ, Sumner DR. The effect of bone remodeling inhibition by zoledronic acid in an animal model of cartilage matrix damage. Osteoarthritis Cartilage 2002;10:226-33.

10. Carbone LD, Nevitt MC, Wildy K, Barrow KD, Harris F, Felson D, et al. The relationship of antiresorptive drug use to structural findings and symptoms of knee osteoarthritis. Arthritis Rheum 2004;50:3516-25

11. Spector TD, Conaghan PG, Buckland-Wright JC, Garnero P, Cline GA, Beary JF, et al. Effect of risedronate on joint structure and symptoms of knee osteoarthritis: results of the BRISK randomized, controlled trial [ISRCTN01928173]. Arthritis Res Ther 2005: 7:R625-33.

12. Bingham CO 3rd, Buckland-Wright JC, Garnero P, Cohen SB, Dougados M, Adami S, et al. Risedronate decreases biochemical markers of cartilage degradation but does not decrease symptoms or slow radiographic progression in patients with medial compartment osteoarthritis of the knee: results of the two-year multinational knee osteoarthritis structural arthritis study. Arthritis Rheum 2006;54:3494-507.

13. Buckland-Wright JC, Messent EA, Bingham CO 3rd, Ward RJ, Tonkin C. A 2 year longitudinal radiographic study examining the effect of a bisphosphonate (risedronate) upon subchondral bone loss in osteoarthritic knee patients. Rheumatology (Oxford) 2007; 46:257-64.

14. Neogi T, Nevitt MC, Ensrud KE, Bauer D, Felson DT. The effect of alendronate on progression of spinal osteophytes and disc space narrowing. Ann Rheum Dis 2008;67:1427-30.

15. Lenart BA, Lorich DG, Lane JM. Atypical fractures of the femoral diaphysis in postmenopausal women taking alendronate. 2008;358:1304-06.

\section{Drug and Therapeutics Bulletin (DTB)}

\section{Your key source of unbiased, independent advice}

For over 45 years DTB has been an independent, indispensable part of evidence-based clinical practice. DTB offers healthcare professionals detailed assessment of, and practical advice on, individual medicines and other treatments, groups of treatment and the overall management of disease.

DTB is now also available online at http://dtb.bmj.com:

- browse or search all DTB content from the latest issue back to 1994

- email alerting, sophisticated searching, RSS feeds and full text links from cited references

- interactive services such as My Folders for quick access to articles that you have viewed previously and My Searches to save and re-use useful searches

- comment online on any DTB article

To subscribe, or for further information, please visit http://dtb.bmj.com 